\title{
Top quark properties and mass measurements with ATLAS
}

\author{
T. Dado ${ }^{\mathrm{a}, \mathrm{b}}$, On behalf of the ATLAS Collaboration \\ ${ }^{a}$ Fakulta matematiky, fyziky a informatiky Univerzity Komenského, Mlynská dolina F1, 84248 Bratislava, Slovakia \\ ${ }^{b}$ Georg-August-Universität Göttingen, II. Physikalisches Institut, Friedrich-Hund-Platz 1, 37077 Göttingen, Germany
}

\begin{abstract}
The top quark plays a unique role among other known quarks as it decays before it forms hadronic bound states. Thus, studying its properties provides an interesting opportunity to access properties of a bare quark directly. The latest measurements of these properties with the ATLAS detector [1] at the LHC are presented. Measurements sensitive to the $W t b$ vertex are summarized and compared to the best theoretical calculations. Effects of colour reconnection on the jet variables in top quark events are presented. Limits on the flavour-changing neutral currents are discussed. The latest top quark decay width and mass measurements are reported.
\end{abstract}

Keywords: top quark, top-quark properties, top-quark mass

\section{Introduction}

With the mass around $173 \mathrm{GeV}$, the top quark is the heaviest known elementary particle. Due to its large mass, the top quark in the Standard Model (SM) has a very large decay width which results in a very short mean life-time of around $0.5 \times 10^{-24} \mathrm{~s}$. Thus the top quark decays before it can form hadronic bound states. This makes the top quark a unique object to study properties of a pseudo-bare quark. In the SM, the top quark decays almost exclusively into a $W$ boson and a $b$ quark. Based on the subsequent decays of the $W$ boson, the three decay channels of $t \bar{t}$ pairs exist: dilepton channel where both $W$ bosons decay leptonically into a charged lepton and the neutrino, lepton+jets channel where one $W$ bosons decays leptonically, the other decays hadronically into two quarks, and the all-hadronic channel where both $W$ boson decay hadronically. Currently, top quarks are produced abundantly at the LHC which allows for precision measurements of its properties.

\footnotetext{
*Talk given at 18th International Conference in Quantum Chromodynamics (QCD 18), 2 July - 6 July 2018, Montpellier - FR

Email address: tomas.dado@cern.ch (T. Dado)
}

\section{Measurement of top quark spin observables}

In the SM, top quarks are produced unpolarised, which means their spins are not aligned with any predefined direction. However, the spins of top and anti top quark are correlated. The magnitude of the correlation depends on the quantizations axes and on the production process. Because of the extremely short mean life-time of top quarks, they decay before any spin-flip can occur [2] and thus transfer all the information about the spin correlations into their decay products.

ATLAS measures the full spin density matrix in events recorded at a centre-of-mass energy of $\sqrt{s}=$ $8 \mathrm{TeV}$ [3]. The measurement uses $t \bar{t}$ pair events in the dileptonic channel. Due to the presence of two neutrinos in the dileptonic channel, the reconstruction of the $t \bar{t}$ pair kinematics is challenging. The neutrino weighting technique $[4,5]$ has been used to fully reconstruct the top quark kinematics. The normalised differential cross-section for $t \bar{t}$ can be expressed as

$$
\begin{aligned}
\frac{1}{\sigma} \frac{d^{2} \sigma}{d \cos \theta_{+}^{a} d \cos \theta_{-}^{b}} & =\frac{1}{4}\left(1+B_{+}^{a} \cos \theta_{+}^{a} B_{-}^{b} \cos \theta_{-}^{b}\right) \\
& -\frac{1}{4} C(a, b,) \cos \theta_{+}^{a} \cos \theta_{-}^{b}
\end{aligned}
$$

where $B^{a}, B^{b}$ and $C(a, b)$ are the polarisation and spin correlations along the spin quantisation axes, $a$ and $b$. 
The angles $\theta^{a}$ and $\theta^{b}$ are defined as angles between the momentum direction of a top-quark decay particle in its parent top-quark's rest frame and the axis $a$ or $b$, respectively. Three different quantization axes are compared: the helicity axis $(k)$ - defined as the top-quark direction in the $t \bar{t}$ rest frame, the transverse axis $(n)$ - defined to be transverse to the production plane created by the topquark direction and the beam axis and the $r$-axis $(r)$ - the axis orthogonal to the other two axes. Obtained results at parton level are

$$
\begin{array}{rlr}
B_{+}^{k} & =-0.044 \pm 0.038[ \pm 0.027 \text { (mass) }], \\
B_{-}^{k} & =-0.064 \pm 0.040[ \pm 0.027 \text { (mass) }], \\
C(k, k) & =0.296 \pm 0.093[ \pm 0.037 \text { (mass) }],
\end{array}
$$

where $+(-)$ denotes decay products of the top (antitop) quark. Dominant systematic uncertainties originate from uncertainty on $t \bar{t}$ modelling. The obtained results are in good agreement with the SM prediction.

\section{Measurement of colour flow in $t \bar{t}$ events}

Although quarks and gluons are produced abundantly at the LHC, due to the confinement only colour-singlet hadrons can be measured directly. High-energy quarks and gluons are measured as jets. The colour connection between partons thus affects the structure of jets. Energy distributions of particles within and between the jets are affected by colour reconnection. The observable that encodes colour information is the jet-pull vector $\overrightarrow{\mathcal{P}}$, defined for a given jet $\mid$ as

$$
\overrightarrow{\mathcal{P}}(\mid)=\sum_{i \in \mathcal{J}} \frac{\left|\overrightarrow{\Delta r}_{i}\right| \cdot p_{T}^{i}}{p_{T}^{\mathcal{T}}} \overrightarrow{\Delta r_{i}},
$$

where $p_{T}^{i}$ is the transverse momentum of a jet constituent $i$ and $\overrightarrow{\Delta r_{i}}=\left(\Delta y_{i}, \Delta \Phi_{i}\right)$ is the offset of the constituent from the jet axis in a rapidity-azimuth plane.

The information about colour reconnection is useful for tuning of Monte Carlo (MC) generators and can also be exploited as a discriminating variable e.g. in the $t \bar{t} H$ process. Top quark pair events in the lepton+jets channel provide a unique opportunity to study the colour reconnection as both colour-connected jets (from the $W$ decay) and colour-disconnected jets (from top quark decays) are present in one event.

Colour connection is measured by ATLAS in data collected in 2015 and 2016 at a centre-of-mass energy of $\sqrt{s}=13 \mathrm{TeV}$ [6] in lepton+jets $t \bar{t}$ events. The measurement investigates tracks matched to jets. Data distributions are unfolded to stable particle level using the Iterative Bayesian technique [7]. Three different observables are compared to MC predictions: 1) the jet pull vector magnitude, the magnitude of the vector defined in eqn. (1), 2) the jet connection vector $\vec{C}\left(\mathcal{J}_{1}, \mathcal{J}_{2}\right)=\overrightarrow{\mathcal{J}}_{1}-$ $\overrightarrow{\mathcal{J}}_{2}$, where $\overrightarrow{\mathcal{J}}_{i}$ is the three-momentum of the jet $i$, and 3 ) the jet pull angle $\Theta_{p}\left(\mathcal{J}_{1} \cdot \mathcal{J}_{2}\right)=\angle\left(\vec{P}\left(\mathcal{J}_{1}\right), \vec{C}\left(\mathcal{J}_{1}, \mathcal{J}_{2}\right)\right)$.

The data shows a weaker effect of the colour connection than all MC simulated samples as illustrated in Figure 1. An exotic, colour-octet model for the $W$ boson, referred to as "colour-flipped" model is compared with the unfolded data in Figure 2.

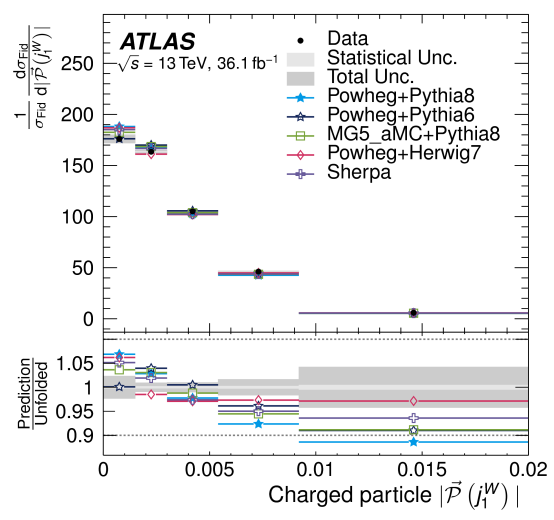

Figure 1: Normalised fiducial differential cross-sections as a function of the magnitude of the jet-pull vector from the leading $W$ boson decay [6]. The data are compared to various SM predictions.

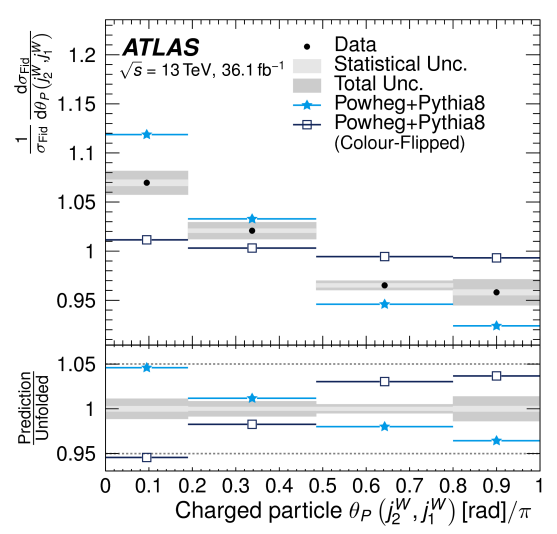

Figure 2: Normalised fiducial differential cross-section as a function of the forward pull angle for the hadronically light (non $b$-tagged) jets from the hadronic decay of the $W$ boson [6]. The data are compared to a SM prediction produced with Powheg+Pythia 8 as well as a model with exotic colour flow also created with Powheg+Pythia 8.

\section{Charge asymmetry}

In proton-antiproton collisions, like at the Tevatron, it is possible to define a forward-backward asymmetry - an angular asymmetry in distributions of top and antitop quarks. At the LHC, where protons are collided 
with protons, the same underlying effect as in protonantiproton collisions induce a charge asymmetry $A_{c}$ defined as

$$
A_{C}=\frac{N(\Delta|y|>0)-N(\Delta|y|<0)}{N(\Delta|y|>0)+N(\Delta|y|<0)},
$$

where $\Delta|y|=\left|y_{t}\right|-\left|y_{\bar{t}}\right|$ is the difference in rapidities of top and antitop quarks and $N(\Delta|y|>0)$ is the number of events with positive $\Delta|y|$, and similarly for $N(\Delta|y|<0)$. In the SM, the charge asymmetry comes from NLO effects [8] and is predicted to be small, however it can be heavily enhanced by some BSM scenarios.

A combination of 7 and $8 \mathrm{TeV}$ measurements of inclusive and differential charge asymmetry from ATLAS and CMS is presented [9]. The combination utilizes the Best Linear Unbiased Estimator (BLUE) method. The combined inclusive charge asymmetry is $A_{C}=$ $0.0055 \pm 0.0023$ (stat.) \pm 0.0025 (syst.), which is in good agreement with the SM prediction. The charge asymmetry is measured in three observables: invariant mass of the $t \bar{t}$ system $\left(m_{t \bar{t}}\right)$, transverse momentum of the $t \bar{t}$ system $\left(p_{T}^{t \bar{t}}\right)$ and longitudinal boost of the $t \bar{t}$ system $(\beta)$. The measurements of the differential charge asymmetry are unfolded to a stable particle level and are combined including bin-by-bin correlations. Figure 3 shows the ATLAS, CMS, and LHC combination for the charge asymmetry as a function of $m_{t \bar{t}}$.

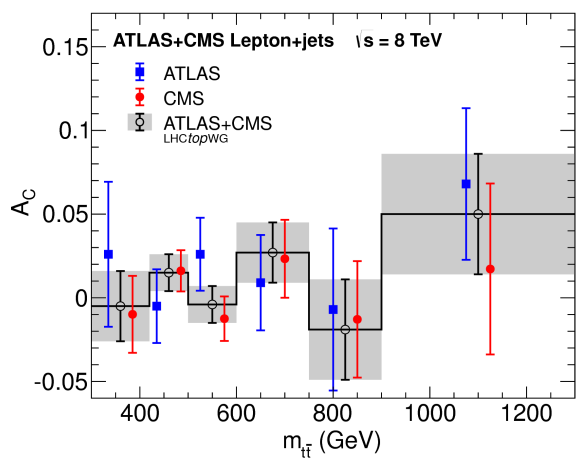

Figure 3: Charge asymmetry in six bins of the invariant mass of the $t \bar{t}$ system as measured in the ATLAS and CMS analyses and the combined results [9]. The last bin includes the overflow. The gray band indicates the uncertainty in the combined result.

\section{Searches for FCNC with top quarks}

Flavour-changing neutral currents (FCNC) are forbidden at tree-level in the SM and are heavily suppressed by the GIM mechanism. Thus, the observation of FCNC processes would hint to BSM physics.

\section{1. $t \rightarrow q H(H \rightarrow \gamma \gamma)$}

ATLAS has performed a search [10] in $t \bar{t}$ events for $t \rightarrow q H(H \rightarrow \gamma \gamma)$, where $q=c, u$ at a centre-of-mass energy of $\sqrt{s}=13 \mathrm{TeV}$ with a dataset corresponding to $36.1 \mathrm{fb}^{-1}$. Events are split into two categories, one targeting the fully hadronic decay of the non-FCNC top quark and the other one targeting the semileptonic decay of the non-FCNC top quark. Events are required to have a diphoton mass $100<m_{\gamma \gamma}<160 \mathrm{GeV}$. Both categories of events are further split into two complementary categories. In the first category, events are required to pass $120<m_{j j j}<220 \mathrm{GeV}$ for the hadronic selection and $130<m_{j \ell v}<210 \mathrm{GeV}$ for the semileptonic selection. The second category consists of events passing all the selection criteria but failing the top mass criterion. Control regions are used to constrain dominant backgrounds: associated production of top-quark pair with a vector boson and diboson processes. No significant excess of events is observed, and limits on the FCNC branching ratios are set: $\operatorname{BR}(t \rightarrow c H)<2.2 \times 10^{-3}$ and $\operatorname{BR}(t \rightarrow u H)<2.4 \times 10^{-3}$ at $95 \% \mathrm{CL}$, assuming $m_{H}=125.09 \mathrm{GeV}$. The precision of the measurement tests regions sensitive to flavour violating Yukawa couplings in two-higgs doublet models (2HDM).

\section{2. $t \rightarrow q H(H \rightarrow$ multilepton $)$}

A search for a top quark decaying into a Higgs boson, $t \rightarrow q H(H \rightarrow$ multilepton $)$, in $t \bar{t}$ events has been conducted by ATLAS [11] at a centre-of-mass energy of $\sqrt{s}=13 \mathrm{TeV}$. The measurement targets multiple decays of the Higgs boson: decays into two $W$ bosons, decays into two $Z$ bosons and decays into two $\tau$ leptons. Only leptonically decaying $\tau$ leptons are considered, hadronically decaying taus are vetoed. Two signal regions are defined: 1) a region with two charged leptons (electrons and muons) with the same sign of electric charge, one $b$-jet, three light jets and two neutrinos and 2) a region with three charged leptons (electrons and muons), one $b$-jet, one light jet and three neutrinos. The signal regions are dominated by the $H \rightarrow W W^{*}$ process. Dominant backgrounds originate from misidentified and non-prompt leptons (fake leptons), associated production of $t \bar{t}$ with a vector boson and diboson processes. A Boosted Decision Tree (BDT) has been trained to discriminate the FCNC signal from the backgrounds. No significant excess of events is observed and limits on the branching ratios are provided: $\mathrm{BR}(t \rightarrow c H)<0.16 \%$ and $\mathrm{BR}(t \rightarrow u H)<0.19 \%$ at 95\% CL. Dominant systematic uncertainties arise from limited statistics for the estimation of fake leptons and diboson normalisation uncertainty. 


\section{3. $t \rightarrow q Z(Z \rightarrow \ell \ell)$}

ATLAS has performed a search [12] for the $t \rightarrow$ $q Z(Z \rightarrow \ell \ell)$ FCNC process in $t \bar{t}$ events at a centre-ofmass energy of $\sqrt{s}=13 \mathrm{TeV}$. The measurement has been performed in the trilepton channel, where the $W$ boson from the non-FCNC top quark decays leptonically into a charged lepton (electron or muon) and the corresponding neutrino, $W \rightarrow \ell v$. The kinematics of the top quarks are reconstructed by minimizing the $\chi^{2}$ formula that takes into account energy conservation and mass constraints from the top quarks and $W$ bosons. Events are required to have $\left|m_{j_{1} \ell_{1} \ell_{2}}-172.5 \mathrm{GeV}\right|<$ $40 \mathrm{GeV},\left|m_{j_{2} \ell_{3} v}-172.5 \mathrm{GeV}\right|<40 \mathrm{GeV}$ and $\mid m_{\ell_{3} v}-$ $80.4 \mathrm{GeV} \mid<30 \mathrm{GeV}$. Dominant backgrounds are diboson processes, associated production of $t \bar{t}$ and a $Z$ boson, associated production of single top and a $Z$ boson and QCD fakes. Five control regions are used to constrain the normalization of the backgrounds in a profile likelihood fit. No significant excess of events is observed and limits on the branching ratios are set: $\mathrm{BR}(t \rightarrow u Z)<1.7 \times 10^{-4}$ and $\mathrm{BR}(t \rightarrow c Z)<2.4 \times 10^{-4}$ at $95 \% \mathrm{CL}$.

Figure 4 summarizes various FCNC searches with top quarks in ATLAS and CMS and compares the obtained results with predictions from some BSM models.

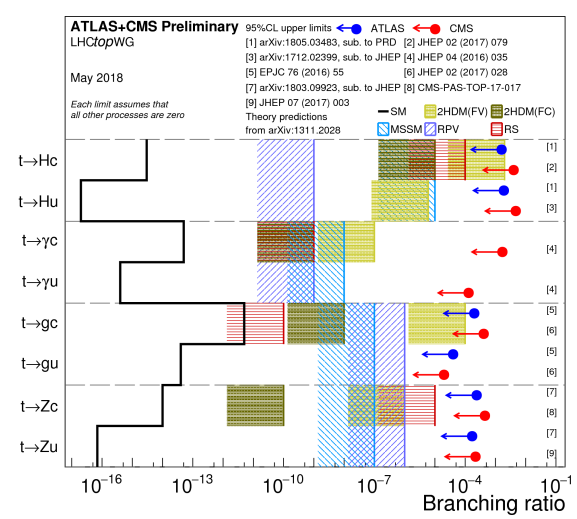

Figure 4: Summary of the current $95 \%$ confidence level observed limits on the branching ratios of the top quark decays via flavour changing neutral currents to a quark and a neutral boson $t \rightarrow X q(X=g, Z$, or $H ; q=u$ or $c$ ) by the ATLAS and CMS Collaborations compared to several new physics models [13].

\section{Top quark decay width measurement}

The first direct measurement of the top-quark decay width by ATLAS has been conducted in the lepton + jets channel of $t \bar{t}$ pair events at a centre-of-mass energy of $\sqrt{s}=8 \mathrm{TeV}$. Events are split by lepton flavour (electrons and muons), $b$-tag multiplicity ( $=1$ and $\geq 2 b$-tagged jets) and by jet $|\eta| \leq 1$ and $|\eta|>1$ to decrease the impact of jet energy uncertainties. The top quark kinematics are reconstructed using the Kinematic Likelihood Fitter (KLFitter) [14]. Two uncorrelated observables are used: $m_{\ell b}$ - the invariant mass of the charged lepton and corresponding $b$-jet and $\Delta R_{\min }\left(j_{b}, j_{l}\right)$ - the minimum $\Delta R$ between the $b$-jet from the hadronically decaying top quark and the closest light jet. Templates corresponding to various top-quark decay widths are created and fitted to the observed data. The resulting top-quark decay width is: $\Gamma_{t}=1.76 \pm 0.33$ (stat.) ${ }_{-0.68}^{+0.79}$ (syst.) $\mathrm{GeV}$. The obtained value is in good agreement with the SM prediction, $\Gamma_{t}=1.32 \mathrm{GeV}$ for a top-quark mass $m_{t}=$ $172.5 \mathrm{GeV}$ [15]. The measurement is dominated by systematic uncertainties: jet energy scale, jet energy resolution and signal modelling uncertainties.

\section{Top-quark mass measurements}

The top-quark mass is one of the fundamental parameters of the SM. Loop corrections relate top-quark mass, Higgs boson mass and $W$ boson masses. A precise measurement of the top-quark mass provides a unique opportunity to test SM.

\subsection{All-hadronic channel}

The top-quark mass has been measured by ATLAS in the all-hadronic $t \bar{t}$ channel [16] at a centre-of-mass energy of $\sqrt{s}=8 \mathrm{TeV}$. Although the all-hadronic channel benefits from the highest branching ratio of all $t \bar{t}$ decays, the measurement is difficult due to an overwhelming multi-jet background. To reduce the contribution of the multi-jet background, events are required to have at least five jets with $p_{T}>60 \mathrm{GeV}$ and the two jets with the highest $b$-tagging weight are required to pass $\Delta \phi\left(b_{1}, b_{2}\right)>1.5$. The remaining multi-jet background is estimated using the ABCD method. The kinematics of the $t \bar{t}$ system are reconstructed using a $\chi^{2}$ technique. The observable sensitive to the top-quark mass is the ratio of the three-jet mass and the two-jet mass, $R_{3 / 2}$. Templates for various discrete values of the top-quark mass are generated and parametrized using Novosibirsk and Landau functions. Due to the correlation between two $R_{3 / 2}$ values originating from the reconstruction, the statistical uncertainty is corrected by a factor of $\sqrt{1+\rho}=1.26$, where $\rho$ is the correlation coefficient. The measured top-quark mass is: $m_{t}=173.72 \pm 0.55$ (stat.) \pm 1.01 (syst.) $\mathrm{GeV}$, which corresponds to a relative uncertainty of $0.7 \%$. Dominant systematic uncertainties arise from the jet energy reconstruction uncertainties. 


\subsection{Lepton+jets channel}

The lepton+jets topology of $t \bar{t}$ events has been exploited by ATLAS [17] to measure the top-quark mass at a centre-of-mass energy of $\sqrt{s}=8 \mathrm{TeV}$. The measurement uses a three dimensional template method. Three dimensions allow for simultaneous measurements of the top-quark mass, the in-situ measurement of the JES and the in-situ measurement of the relative $b$-jet-to-light jet JES. The templates have been created from the reconstructed top-quark mass $m_{t}^{\text {reco }}, m_{W}^{\text {reco }}$ and $R_{b q}^{\text {reco }}=\frac{p_{T}^{b_{\text {had }}}+p_{T}^{b_{\text {lep }}}}{p_{T}^{q_{1}}+p_{T}^{q_{2}}}$ - a scalar sum of the transverse momenta of the two $b$-tagged jets divided by the scalar sum of the transverse momenta of two jets associated with the hadronic $W$ decay. KLFitter has been used to identify jets originating from the decays of the top quarks. To further improve the performance of the identification, a reconstruction BDT has been trained with the KLFitter output as an input for the BDT. The evolution of the statistical, dominant systematic and total uncertainties has been checked in order to find the optimal cut on the BDT output to minimize the total uncertainty on the top-quark mass. The templates have been fitted to data and the resulting top-quark mass is: $m_{t}=172.08 \pm 0.39$ (stat.) \pm 0.82 (syst.) GeV, which corresponds to a relative uncertainty of $0.5 \%$. Dominant systematic uncertainties arise from the JES and the $b$ tagging uncertainties.

\subsection{Pole-mass measurement}

Differential cross section measurements with fixedorder predictions correspond more directly to the polemass. ATLAS has performed a measurement [18] of the top-quark pole-mass in dilepton $t \bar{t}$ events at a centre-ofmass energy of $\sqrt{s}=8 \mathrm{TeV}$. Some of the differential cross-sections of leptonic observables are sensitive to the top-quark mass. Measured distributions are compared to predictions from both NLO plus parton shower event generators and fixed-order QCD calculations. The former are similar to traditional MC mass measurements but are less sensitive to hadronization modelling uncertainties as they rely purely on leptonic observables. In the measurement of $m_{t}^{\text {pole }}$ from fixed-order QCD predictions, $\chi^{2}$ values are calculated for comparison of data to predictions with different $m_{t}^{\text {pole }}$ and the best-fit $m_{t}^{\text {pole }}$ is found by polynomial interpolation. The $m_{t}^{\text {pole }}$ value used in the fixed-order predictions corresponds to a well-defined renormalisation scheme, which is the pole-mass scheme within the MCFM implementation. Combination of eight lepton distributions yields: $m_{t}^{\text {pole }}=173.2 \pm 0.9$ (stat.) \pm 0.8 (syst.) \pm 1.2 (theory) $\mathrm{GeV}$, which corresponds to a relative uncertainty of $1 \%$. The dominant systematic uncertainty originates from an uncertainty of the choice of the functional form of QCD scales.

Figure 5 summarizes various top-quark mass measurements performed by ATLAS and CMS as well as their combinations. It should be noted that even though individual ATLAS top-quark mass measurements have larger total uncertainties than measurements conducted by CMS, due to large correlation of systematic uncertainties in the combination, the combined ATLAS results have very similar total uncertainties as the combined CMS results.

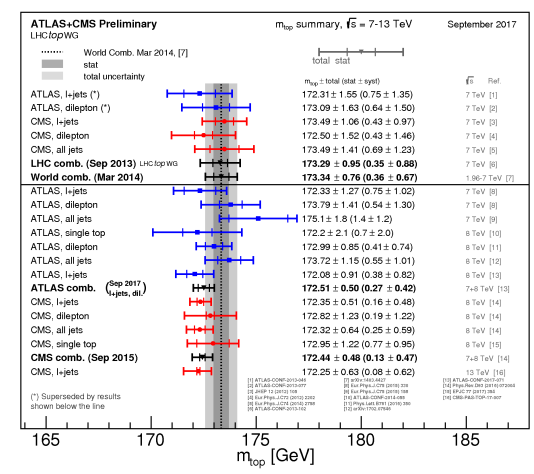

Figure 5: Summary of the ATLAS and CMS direct $m_{t}$ measurements [13]. The results are compared with the LHC and Tevatron $+\mathrm{LHC} m_{t}$ combinations. The results below the line are results produced after the LHC and the Tevatron+LHC combinations were performed.

\section{Conclusions}

This proceeding contribution reviewed some of the recent results on top-quark mass and top-quark properties measurements performed by the ATLAS collaboration with the data collected at the LHC. Measurements of top-quark spin observables, colour connection, charge asymmetry, top-quark decay width, and top-quark mass are summarized. Furthermore, searches for FCNC processes with the top quark are presented.

Copyright 2018 CERN for the benefit of the ATLAS Collaboration. Reproduction of this article or parts of it is allowed as specified in the CC-BY-4.0 license.

\section{References}

[1] ATLAS Collaboration, JINST 3, S08003 (2008)

[2] A. F. Falk, M. E. Peskin, Phys. Rev. D 49, 3320-3332 (1994) 
[3] ATLAS Collaboration, JHEP 03, 113 (2017)

[4] D0 Collaboration, Phys. Rev. Lett. 80, 2063-2068 (1998)

[5] ATLAS Colaboration, JHEP 05, 061 (2015)

[6] ATLAS Collaboration, arXiv:1805.02935 (Submitted to EPJC)

[7] G. D’Agostini, NIM A 362, 487-498 (1995)

[8] J. H. Kühn, G. Rodrigo, JHEP 01, (2012) 63

[9] ATLAS and CMS Collaborations, JHEP 04 (2018) 033

[10] ATLAS Collaboration, JHEP 10 (2017) 129

[11] ATLAS Collaboration, arXiv:1805.03483 (Submitted to PRD)

[12] ATLAS Collaboration, arXiv:1803.09923 (Submitted to JHEP)

[13] ATLAS Collaboration, ATLAS Top summary plots, https: //twiki.cern.ch/twiki/bin/view/AtlasPublic/ TopPublicResults

[14] Johannes Erdmann et. al, NIM A 748, 18-25 (2014)

[15] J. Gao, C. S. Li and H. X. Zhu, Phys. Rev. Lett. 110, 042001 (2013)

[16] ATLAS Collaboration, JHEP 09 (2017) 118

[17] ATLAS Collaboration, ATLAS-CONF-2017-071, https:// cds.cern. ch/record/2285809

[18] ATLAS Collaboration, Eur. Phys. J. C 77 (2017) 804 